\title{
Computing Distinguishing Formulas for Branching Bisimulation
}

\author{
Henri Korver \\ Department of software technology, CWI \\ P.O. Box 4079, 1009 AB Amsterdam, The Netherlands \\ e-mail: henri@cwi.nl
}

\begin{abstract}
Branching bisimulation is a behavioral equivalence on labeled transition systems which has been proposed by Van Glabbeek and Weijland as an alternative to Milner's observation equivalence. This paper presents an algorithm which, given two branching bisimulation inequivalent finite state processes, produces a distinguishing formula in Hennessy-Milner logic extended with an 'until' operator. The algorithm, which is a modification of an algorithm due to Cleaveland, works in conjunction with a partition-refinement algorithm for deciding branching bisimulation equivalence. Our algorithm provides a useful extension to the algorithm for deciding equivalence because it tells a user why certain finite state systems are inequivalent.
\end{abstract}

Note: The research of the author is supported by the European Communities under RACE project no. 1046, Specification and Programming Environment for Communication Software (SPECS). This article does not necessarily reflect the view of the SPECS project.

\section{Introduction}

The complexity of concurrent systems (parallel chips, computer networks) is still increasing every day. To cope with this problem a lot of research has been spent on the development of formal verification techniques that guarantee the reliability of these systems.

At the moment, the use of behavioral equivalences is considered as a promising approach towards system verification. In this approach, concurrent systems are modeled as transition graphs, and verification amounts to establishing that the graph representing the implementation of the system is equivalent to (behaves the same as) the graph representing the specification of the system. The main advantage of this approach is that behavioral equivalences can be decided fully automatically on finite transition graphs and that several equivalences can be decided efficiently.

A number of equivalences have been proposed in the literature [3], and several automated tools include facilities for computing them [8]. One particularly interesting equivalence is (strong) bisimulation equivalence [10], which serves as the basis for a number of other equivalences that can be described in terms of it. Bisimulation equivalence has a logical characterization: two systems are equivalent exactly when they satisfy the 
same formulas in a simple modal logic due to Hennessy and Milner [6]. This fact suggests a useful diagnostic methodology for tools that compute bisimulation equivalence: when two systems are found not to be equivalent, one may explain why by giving a (distinguishing) formula satisfied by one and not by the other.

Recently, Cleaveland developed an advanced technique to generate such distinguishing formulas automatically. His method works in conjunction with the efficient partitionrefinement algorithm for computing bisimulation equivalence and is described in [1]. The formulas generated by this algorithm are often minimal in a precisely defined sense.

In this paper, we apply this technique to branching bisimulation equivalence [4] which is a more suitable for practical purposes than (strong) bisimulation equivalence. Branching bisimulation equivalence resembles the well-known observation equivalence [10] and can be decided more efficiently [5].

Branching bisimulation is characterized in terms of Hennessy-Milner Logic with an Until-operator (HMLU) [2] and we develop a technique for determining a HMLU-formula that distinguishes two branching bisimulation inequivalent finite-state systems, using the idea of the advanced method of Cleaveland. To this end, we show how to use information generated by an adapted version of the partition-refinement algorithm of Groote and Vaandrager [5] to compute such a formula efficiently. On the basis of this result, tools using branching bisimulation may be modified to give users diagnostic information in the form of a distinguishing formula when a system is found not to be equivalent to its specification.

The remainder of the paper is organized as follows. The next section defines branching bisimulation equivalence and examines the connection between it and the HennessyMilner Logic with Until. Section 3.1 describes the algorithm of Groote and Vaandrager to compute branching bisimulation equivalence on the states of a transition graph. Then section 3.2 describes how to generate a block tree which retains information computed by the equivalence algorithm. Finally, in section 3.3 it is shown how to compute distinguishing formulas on the basis of this block tree; a small example is also presented to illustrate the working of the new algorithm.

For proofs, the reader is referred to the full version of this paper [9].

\section{Acknowledgements}

In the first place, I would like to thank Rance Cleaveland for writing his article and for answering all my questions. Special thanks to Jan Friso Groote for reading the previous draft of this paper very carefully.

\section{Transition Graphs, Branching Bisimulation and HMLU}

Concurrent systems are often modeled by transition graphs. Vertices in these graphs correspond to the states a system may enter as it executes, with one vertex being distinguished as the start state. The edges, which are directed, are labeled with actions and represent the state transitions a system may undergo. The formal definition is the following. 
Definition 2.1 A labeled transition graph is a quadruple $\langle S, s$, Act, $\rightarrow>$, where:

- $S$ is a set of states;

- $s \in S$ is the start state;

- Act is a set of actions; the silent action $\tau$ is not in Act; and

- $\rightarrow \subseteq S \times A c t_{\tau} \times S$ is the transition relation where Act $_{\tau}=A c t \cup\{\tau\}$. An element $(p, \alpha, q) \in \rightarrow$ is called a transition, and is usually written as $p \stackrel{\alpha}{\longrightarrow} q$.

The silent action $\tau$ is unobservable for the environment and is used to symbolize the internal behavior of the system.

When a graph does not have a start state indicated, we shall refer to the corresponding triple as a transition system. A state in a transition system gives rise to a transition graph in the obvious way: let the given state be the start state, with the three components of the transition graph coming from the transition system.

Transition graphs are often too concrete for representing concurrent systems. Mostly one is only interested in the observational behavior of a complicated system and one is not interested in the internal (low-level) computations. Branching bisimulation, which is an interesting alternative for the well-known observation equivalence [10], remedies this shortcoming. In [4] several definitions of branching bisimulation are given, which all lead to the same equivalence. The following definition is in our setting most suitable.

\section{Definition 2.2 (Branching bisimulation)}

- Let $<S$, Act, $\rightarrow>$ be a transition system. A relation $R \subseteq S \times S$ is called a branching bisimulation if it is symmetric and satisfies the following transfer property:

If $r R s$ and $r \stackrel{\alpha}{\longrightarrow} r^{\prime}$, then either $\alpha=\tau$ and $r^{\prime} R s$ or; $\exists s_{0}, . ., s_{n}, s^{\prime} \in S: s=s_{0}$, $\left[\forall_{0<i \leq n}: s_{i-1} \stackrel{\tau}{\longrightarrow} s_{i}\right]$ and $s_{n} \stackrel{\alpha}{\longrightarrow} s^{\prime}$ such that $\forall_{0<i \leq n} r R s_{i}$ and $r^{\prime} R s^{\prime}$.

- Two states $r$ and $s$ are branching bisimilar, abbreviated $r \approx_{B} s$ or $s \approx_{B} r$, if there exists a branching bisimulation relating $r$ and $s$.

The arbitrary union of branching bisimulation relations is again a branching bisimulation; $\approx_{B}$ is the maximal branching bisimulation and is an equivalence relation.

Let $T_{1}=<S_{1}, s_{1}, A c t, \rightarrow_{1}>$ and $T_{2}=<S_{2}, s_{2}, A c t, \rightarrow_{2}>$ be two transition graphs satisfying $S_{1} \cap S_{2}=\emptyset$. Then $T_{1}$ and $T_{2}$ are branching bisimilar exactly when the two start states, $s_{1}$ and $s_{2}$ are branching bisimilar in the transition graph $<S_{1} \cup S_{2}, A c t, \rightarrow_{1}$ $\cup \rightarrow_{2}>$.

Branching bisimulation has a logical characterization in terms of the Hennessy-Milner Logic with Until (HMLU): two states are equivalent exactly when they satisfy the same set of HMLU-formulas (see [2]). In [2] also two other logics are given that characterize branching bisimulation. We think that HMLU is the most natural choice in this setting. HMLU is a simple modal logic; the syntax of formulas is defined by the following grammar, where $\alpha \in A c t_{\tau}$.

$$
\Phi::=t t|\neg \Phi| \Phi \wedge \Phi \mid \Phi\langle\alpha\rangle \Phi
$$

The semantics of the logic is given with respect to a transition system $\mathrm{T}=\langle\mathrm{S}, \mathrm{Act}, \rightarrow\rangle$ 


$$
\begin{aligned}
\llbracket t t \rrbracket_{T} & =S \\
\llbracket \neg \Phi \rrbracket_{T}= & S-\llbracket \Phi \rrbracket_{T} \\
\llbracket \Phi_{1} \wedge \Phi_{2} \rrbracket_{T}= & \llbracket \Phi_{1} \rrbracket_{T} \cap \llbracket \Phi_{2} \rrbracket_{T} \\
\llbracket \Phi_{1}\langle\alpha\rangle \Phi_{2} \rrbracket_{T}= & \left\{s \in S \mid\left(\alpha=\tau \text { and } s \in \llbracket \Phi_{2} \rrbracket_{T}\right)\right. \text { or } \\
& \left(\exists s_{0}, \ldots, s_{n} \in \llbracket \Phi_{1} \rrbracket_{T}, \exists s^{\prime} \in \llbracket \Phi_{2} \rrbracket_{T}: s_{0}=s\right. \\
& \left.\left.\left.\quad \forall_{0<i \leq n}: s_{i-1} \stackrel{\tau}{\longrightarrow} s_{i}\right] \text { and } s_{n} \stackrel{\alpha}{\longrightarrow} s^{\prime}\right)\right\}
\end{aligned}
$$

Figure 1: The semantics of formulas in Hennessy-Milner Logic with Until.

and appears in Figure 1. In this figure each formula is mapped to the set of states for which the formula is 'true'. We shall omit explicit reference to the transition system used to interpret formulas when it is clear from the context. Intuitively, the formula $t t$ holds in any state, and $\neg \Phi$ holds in a state if $\Phi$ does not. The formula $\Phi_{1} \wedge \Phi_{2}$ holds in a state if both $\Phi_{1}$ and $\Phi_{2}$ do. The until-proposition $\Phi_{1}\langle\alpha\rangle \Phi_{2}$ holds in a state, if this state can reach via $\alpha$, a state in which $\Phi_{2}$ holds while moving through intermediate states in which $\Phi_{1}$ holds.

Let $\mathcal{H}(s)$ be the set of HMLU-formulas that are valid in state $s$ :

$$
\mathcal{H}(s)=\{\Phi \mid s \in \llbracket \Phi \rrbracket\} .
$$

The next theorem is a specialization of a theorem proved in [2].

Theorem 2.3 Let $\left\langle S\right.$, Act, $\rightarrow>$ be a finite-state transition system, with $s_{1}, s_{2} \in S$. Then $\mathcal{H}\left(s_{1}\right)=\mathcal{H}\left(s_{2}\right)$ if and only if $s_{1} \approx_{B} s_{2}$.

It follows that if two states in a (finite-state) transition system are inequivalent, then there must be a HMLU-formula satisfied by one and not the other. This is the basis of our definition for distinguishing formula, although we shall in fact use the following, slightly more general formulation taken from [1].

Definition 2.4 Let $\left\langle S, A c t, \rightarrow>\right.$ be a transition system, and let $S_{1} \subseteq S$ and $S_{2} \subseteq S$. Then $H M L U$-formula $\Phi$ distinguishes $S_{1}$ from $S_{2}$ if the following hold.

$$
\begin{aligned}
& \text { 1. } S_{1} \subseteq \llbracket \Phi \rrbracket . \\
& \text { 2. } S_{2} \cap \llbracket \Phi \rrbracket=\emptyset .
\end{aligned}
$$

So $\Phi$ distinguishes $S_{1}$ from $S_{2}$ if every state in $S_{1}$, and no state in $S_{2}$, satisfies $\Phi$. Theorem 2.3 thus guarantees the existence of a formula that distinguishes $\left\{s_{1}\right\}$ from $\left\{s_{2}\right\}$ if $s_{1} \not{ }_{B} s_{2}$.

Finally, we take the following criterion from [1] to indicate whether a distinguishing formula contains extraneous information.

Definition 2.5 Let $\Phi$ be a $H M L U$-formula distinguishing $S_{1}$ from $S_{2}$. Then $\Phi$ is minimal if no $\Phi^{\prime}$ obtained by replacing a non-trivial subformula of $\Phi$ with the formula tt distinguishes $S_{1}$ from $S_{2}$. 
Intuitively, $\Phi$ is a minimal formula for $S_{1}$ with respect to $S_{2}$ if each of its subformulas plays a role in distinguishing the two. This notion of minimality is rather naive, but at the moment we are not aware of a better definition.

\section{Computing Distinguishing Formulas}

In this section, we describe a partition refinement algorithm for computing branching bisimulation equivalence and show how to alter it to generate a block tree. Then given such a block tree, we describe how to generate distinguishing formulas. Finally, a small example is given that illustrates the use of the algorithm.

\subsection{Computing Branching Bisimulation}

At the moment, 'partition-refinement' is the most efficient method to compute bisimulation equivalences [7]. A partition-refinement algorithm exploits the fact that an equivalence relation on the set of states may be represented as a partition, or a set of pairwise-disjoint subsets (called blocks) of the state set whose union is the whole state set. In this representation blocks correspond to the equivalence classes, so two states are equivalent exactly when they belong to the same block. Beginning with the partition containing one block (representing the trivial equivalence relation consisting of one equivalence class), the algorithm repeatedly refines a partition by splitting blocks until the associated equivalence relation becomes a bisimulation.

In [5] the refinement strategy to obtain branching bisimulation is described. To refine the current partition, the algorithm of Groote and Vaandrager looks at each block in turn. If a state in block $B$ can reach via action $\alpha$, possibly after some initial stuttering, a state in block $B^{\prime}$ and another state in $B$ does not, then the algorithm splits $B$ into two blocks. The first block contains all the states which can reach via action $\alpha$, possibly after some initial stuttering, a state in block $B^{\prime}$. The second block contains all the other states. When no more splitting is possible, the resulting equivalence corresponds exactly to branching bisimulation on the given transition system.

Below we present the definitions and the algorithm in more formal notation; the description is a slight modification of the one in [5].

\section{Definition 3.1}

Let $\langle S, A c t, \rightarrow\rangle$ be a transition system.

1) A collection $\left\{B_{j} \mid j \in J\right\}$ of nonempty subsets of $S$ is called a partition if $\bigcup_{j \in J} B_{j}=S$ and for $i \neq j: B_{i} \cap B_{j}=\emptyset$. The elements of a partition are called blocks.

2) If $\mathcal{P}$ and $\mathcal{P}^{\prime}$ are partitions of $S$ then $\mathcal{P}^{\prime}$ refines $\mathcal{P}$, if any block of $\mathcal{P}^{\prime}$ is included in a block of $\mathcal{P}$.

3) The equivalence $\sim_{\mathcal{P}}$ on $S$ induced by a partition $\mathcal{P}$ is defined by:

$r \sim \mathcal{P} s \Leftrightarrow \exists B \in \mathcal{P}: r \in B \wedge s \in B$.

4) For $B, B^{\prime}$ we define the set pos ${ }_{\alpha}\left(B, B^{\prime}\right)$ as the set of states in $B$ from which, after some internal $\tau$-stuttering, a state in $B^{\prime}$ can be reached:

$\operatorname{pos}_{\alpha}\left(B, B^{\prime}\right)=\left\{s \in B \mid \exists s_{0}, \ldots, s_{n} \in B, \exists s^{\prime} \in B^{\prime}:\right.$

$$
\left.s_{0}=s,\left[\forall_{0<i \leq n}: s_{i-1} \stackrel{\tau}{\longrightarrow} s_{i}\right] \text { and } s_{n} \stackrel{\alpha}{\longrightarrow} s^{\prime}\right\}
$$

5) We say that $B^{\prime}$ is a splitter of $B$ with respect to action $\alpha$ iff 
$B \neq B^{\prime}$ or $\alpha \neq \tau$, and $\emptyset \neq \operatorname{pos}_{\alpha}\left(B, B^{\prime}\right) \neq B$.

6) If $\mathcal{P}$ is a partition of $S$ and $B^{\prime}$ is a splitter of $B$ with respect to $\alpha$, then $\operatorname{Ref}_{p}^{\alpha}\left(B, B^{\prime}\right)$ is the partition $\mathcal{P}$ where $B$ is replaced by $\operatorname{pos}_{\alpha}\left(B, B^{\prime}\right)$ and $B-\operatorname{pos}_{\alpha}\left(B, B^{\prime}\right)$.

7) $\mathcal{P}$ is stable with respect to block $B^{\prime}$ if for no block $B$ and for no action $\alpha, B^{\prime}$ is a splitter of $B$ with respect to $\alpha . \mathcal{P}$ is stable if it is stable with respect to all its blocks.

Algorithm 3.2 The algorithm to compute branching bisimulation maintains a partition $\mathcal{P}$ that is initially $\mathcal{P}_{0}=\{S\}$. It repeats the following step, until $\mathcal{P}$ is stable:

Find blocks $B, B^{\prime} \in \mathcal{P}$ and a label $\alpha \in$ Act $_{\tau}$ such that $B^{\prime}$ is a splitter wrt. $\alpha$; $\mathcal{P}:=\operatorname{Ref}_{p}^{\alpha}\left(B, B^{\prime}\right)$.

In [5] it is proved that the equivalence induced by the last partition computed by algorithm 3.2 corresponds exactly to branching bisimulation equivalence. Also, the complexity bounds given in [5] are presented.

Theorem 3.3 Let $\langle S$, Act,$\rightarrow\rangle$ be a finite transition system. Let $\mathcal{P}_{f}$ be the final partition obtained by the algorithm above. Then $\sim_{\mathcal{P}_{f}}=\approx_{B}$.

Theorem 3.4 The time complexity of algorithm 3.2 is $O(|S| *|\rightarrow|)$. And the space complexity is $O(|\rightarrow|)$.

\subsection{Generating The Block Tree}

In addition to computing the partition as described above, we now retain information about how and why the blocks are split by construction of a labeled block 'tree'. The following definitions are used to describe the generation procedure of such a block tree.

Definition 3.5 (Parent and its children)

$\mathcal{P}(B)$ is the parent of block $B$ in the block tree. $\mathcal{L}(B)$ is the left child of block $B . \mathcal{R}(B)$ is the right child of block $B$. When $B$ has a single child or no children at all then $\mathcal{L}(B)$ and $\mathcal{R}(B)$ are undefined.

Definition 3.6 (Height of a block)

The height of a block $B$ in the block tree is defined as follows:

$h(B):=0 \quad$ where $B$ is the root block.

$h(B):=1+h(\mathcal{P}(B))$.

\section{Definition 3.7 (Parent Partition)}

The Parent Partition of block $B$ in the block tree, is the partition where $B$ is created. $\mathcal{P} \mathcal{P}(B):=\{C \mid h(C)=h(\mathcal{P}(B))\}$.

Definition 3.8 (Blocks that can be reached from block $B$ )

Let $B$ be a block in the block tree.

- $r_{\alpha}(B):=\left\{C \in \mathcal{P} \mathcal{P}(B) \mid \exists s \in B, s^{\prime} \in C: s \stackrel{\alpha}{\longrightarrow} s^{\prime}\right\} ; r_{\alpha}(B)$ contains all the blocks in the parent partition of $B$ that can be reached from a state in $B$ via $\alpha$.

- $r_{\tau}^{p}(B):=\left\{C \in \mathcal{P} \mathcal{P}(B) \mid \exists s \in B, s^{\prime} \in C: s \stackrel{\tau}{\longrightarrow} s^{\prime} \wedge C \neq \mathcal{P}(B)\right\} ; \quad r_{\tau}^{p}(B)$ contains all the blocks in the parent partition of $B$ that can be reached from a state in $B$ via $\tau$. The superscript ' $p$ ' indicates that the parent of $B$ is not included. 
Algorithm 3.2 is modified as follows. Rather than discarding an old partition after it is refined, the new procedure constructs a tree of blocks as follows. The children of a block are the new blocks that result when the algorithm splits the block; accordingly, the root is labeled with the block $S$, and after each refinement the leaves of this tree represents the current partition.

When a block $P$ is split due to splitter block $B^{\prime}$ and action $\alpha$, we position the new block $L=\operatorname{pos}_{\alpha}\left(P, B^{\prime}\right)$ as the left child and the new block $R=P-p_{\alpha}\left(P, B^{\prime}\right)$ as the right child, and we label the arc connecting $P$ to $L$ with $\alpha$ and $B^{\prime}$. We label the arc connecting $P$ to $R$ with $r_{\tau}^{p}(R)$ and $r_{\alpha}(R)$, these block-sets are given in definition 3.8. The blocks in $r_{\tau}^{p}(R)$ bear witness to the states in $R$ that cannot evolve in internal stuttering. The blocks in $r_{\alpha}(R)$ bear witness to the states in $R$ that cannot reach the splitter block by an $\alpha$-step.

Recall that every state in $L$ can reach via $\alpha$, possibly after some initial stuttering, a state in $B^{\prime}$ and no state in $R$ does. If a block is not split during a refinement, it is assigned a copy of itself as its only child Figure 3 contains an example of such a tree.

The construction of the block tree during the partition-refinement algorithm does not influence the time complexity. The space complexity has changed slightly from $O(|\rightarrow|)$ to $O\left(|S|^{2}\right.$ ) due to the following theorem (note that $\left|\mathcal{P}_{f}\right| \leq|S|$ ).

Theorem 3.9 The space requirement of the labeled block tree is $O\left(|S|+\left|P_{f}\right|^{2}\right)$.

Proof. Strictly speaking, only the leaves in the tree need to be labeled with the corresponding sets of states.

\subsection{Generating Distinguishing Formulas}

Given a block tree computed by the extended partition-refinement algorithm above, and two disjoint blocks $B_{1}$ and $B_{2}$, the following postprocessing step builds a formula $\Delta\left(B_{1}, B_{2}\right)$ that distinguishes the states in $B_{1}$ from those in $B_{2}$.

First we compute the lowest common ancestor of $B_{1}$ and $B_{2}$ (and call it $P$ ). By lemma 3.11 we know that a formula distinguishing the children of $P$, also distinguishes $B_{1}$ from $B_{2}$.

\section{Definition 3.10 (Lowest Common Ancestor)}

The function $\mathcal{L C} \mathcal{A}$ returns the Lowest Common Ancestor of two disjoint blocks $B_{1}$ and $B_{2}$ in the block tree.

Lemma 3.11

$P=\mathcal{L} \mathcal{C} \mathcal{A}\left(B_{1}, B_{2}\right)$

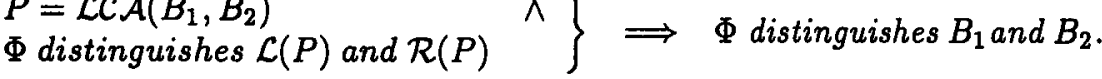

Proof. $\quad B_{1}$ and $B_{2}$ are subsets of respectively $\mathcal{L}(P)$ and $\mathcal{R}(P)$.

For easy notation, let $L=\mathcal{L}(P)$ be the left child and $R=\mathcal{R}(P)$ the right child of $P$. The arc connecting blocks $P$ and $L$ is labeled with $\alpha$ and $B^{\prime} ;$ and the arc connecting blocks $P$ and $R$ is labeled with respectively the block-sets $r_{\tau}^{p}(R)$ and $r_{\alpha}(R)$ (call these block-sets respectively $r_{1}$ and $r_{2}$ ).

From the way that the block tree is generated, we know that every state in $L$ can reach via action $\alpha$, possibly after some initial stuttering, a state in $B^{\prime}$ and that no state in $R$ 
does. Accordingly, one recursively builds formulas that distinguish $P$ and blocks in $r_{1}$, and takes their conjunction (call it $\Phi_{1}$ ). And, if one also recursively builds formulas that distinguish $B^{\prime}$ from each block in $r_{2}$ and also takes their conjunction (call it $\Phi_{2}$ ), then every state in $L$ satisfies $\Phi_{1}\langle\alpha\rangle \Phi_{2}$ (call this formula $\Phi$ ) and no state in $R$ does. In case $\alpha=\tau$, one has to add the extra conjunct $\Delta\left(B^{\prime}, R\right)$, to ensure that $\Phi$ is a distinguishing formula; this is caused by the first disjunct at the right hand side of the last mapping in figure 1 . The details are given below.

\section{Algorithm 3.12}

When $B_{1}$ and $B_{2}$ are disjoint, $\Delta\left(B_{1}, B_{2}\right)$ can be computed recursively as follows.

1. Compute $P:=\mathcal{L C} \mathcal{A}\left(B_{1}, B_{2}\right)$.

2. Let $L:=\mathcal{L}(P) ; R:=\mathcal{R}(P)$.

(Notice that $B_{1} \subseteq L$ and $B_{2} \subseteq R$, or $B_{2} \subseteq L$ and $B_{1} \subseteq R$.)

3. Let $\alpha$ and $B^{\prime}$ be the labels on the arc connecting $P$ and $L$; and

let $r_{1}:=r_{\tau}^{p}(R)$ and $r_{2}:=r_{\alpha}(R)$ be the labels on the arc connecting $P$ and $R$;

- if $r_{1}=\emptyset$ then $\Phi_{1}:=t t$ else $\Phi_{1}:=\bigwedge_{C \in r_{1}} \Delta(P, C)$;

- if $r_{2}=\emptyset$ then $\Phi_{2}:=t$ else $\Phi_{2}:=\bigwedge_{C \in r_{2}} \Delta\left(B^{\prime}, C\right)$.

4. If $\alpha \neq \tau$ then $\Phi:=\Phi_{1}\langle\alpha\rangle \Phi_{2}$.

else $\quad \Phi_{3}:=\Delta\left(B^{\prime}, R\right)$;

$$
\Phi:=\Phi_{1}\langle\tau\rangle\left(\Phi_{2} \wedge \Phi_{3}\right) \text {. }
$$

5. If $B_{1} \subseteq L$ then return $\Phi$ else return $\neg \Phi$.

We now have the following theorem.

Theorem 3.13 $B_{1} \cap B_{2}=\emptyset \Longrightarrow \Delta\left(B_{1}, B_{2}\right)$ distinguishes $B_{1}$ and $B_{2}$.

Proof. By induction on the depth of $B_{1}$ and $B_{2}$ in the block tree.

It should be noted that exponential length formulas may be generated. However, one may present such a formula (as a set of propositional equations) in space proportional to $\left|\mathcal{P}_{f}\right|^{2}$, where $\mathcal{P}_{f}$ is the final partition computed by the algorithm (note that $\left|\mathcal{P}_{f}\right| \leq|S|$ ). This results from the fact that there can be at most $\left|\mathcal{P}_{f}\right|-1$ recursive calls generated by the above procedure and the fact that each distinguishing formula is of the form $(\neg) \Phi_{1}\langle\alpha\rangle \Phi_{2}$, where $\Phi_{1}$ and $\Phi_{2}$ contain together at most $\left|\mathcal{P}_{f}\right|-1$ conjuncts, each of the form $\Delta\left(B_{i}, B_{j}\right)$ for some $B_{i}$ and $B_{j}$.

Theorem 3.14 An equational representation of $\Delta\left(B_{1}, B_{2}\right)$ may be calculated in $O\left(\left|\mathcal{P}_{f}\right|^{2}\right)$ time, once the tree of blocks has been computed.

Proof. At each recursive call, computing the lowest common ancestor requires at most $O\left(\left|\mathcal{P}_{f}\right|\right)$ work.

In general a formula $\Delta\left(s_{1}, s_{2}\right)$ will not be minimal in the sense of definition 2.5. In [1] the following straightforward procedure is proposed to minimize $\Delta\left(s_{1}, s_{2}\right)$ once it has 
been computed. Repeatedly replace subformulas in the formula by $t t$ and see if the resulting formula still distinguishes $s_{1}$ from $s_{2}$. If so, the subformula may either be omitted (if it is one of several conjuncts in a larger conjunction) or left at $t$. The result of this would be a minimal formula. The computational tractability of this procedure remains to be examined.

We close this subsection with a general remark about our method. Our method generates a formula that distinguishes blocks that may contain more than one state, but mostly one is only interested in a formula that distinguishes two particular states. In this case, algorithm 3.12 can also be used to construct a formula distinguishing two inequivalent states $s_{1}$ and $s_{2}$; first locate the disjoint blocks $B_{1}$ and $B_{2}$ such that $s_{i} \in B_{i}(i=1,2)$, then build $\Delta\left(B_{1}, B_{2}\right)$.

\subsection{An Example}

To illustrate our algorithm we consider two transition graphs that are not branching bisimulation equivalent. Figure 2 shows the transition system that includes the two transition graphs. It is interesting to notice that these two graphs are an instance of the second $\tau$-law of observation equivalence (see e.g. [10]); so they are not differentiated by HML without Until-operator. State $s_{1}$ is the start state of one graph, while state $s_{5}$ is the start state of the other. Figure 3 contains a tree of blocks generated by the altered partition-refinement algorithm. Notice that $s_{1} \not \not_{B} s_{5}$, as they are in different blocks. In order to build a formula that $s_{1}$ satisfies and $s_{5}$ does not, it suffices to generate $\Delta\left(B_{6}, B_{5}\right)$, the formula that distinguishes block $B_{6}$ and $B_{5}$. To do so, the algorithm first locates the lowest common ancestor of the two blocks $\left(B_{3}\right.$, in this case). The left child is $B_{5}$ and the right child is $B_{6}$. The labels on the left arc indicate that the action causing the split is $a$, and the splitter block is $B_{2}$. The labels on the right arc indicate that $r_{\tau}^{p}(R)=\left\{B_{4}\right\}$ and $r_{\alpha}(R)=\emptyset$. The formula that will be returned, then, will be

$$
\neg\left(\Delta\left(B_{3}, B_{4}\right)\langle a\rangle t t\right) ;
$$

this formula holds of $s_{1}$ and not of $s_{5}$. By repeating this process, it turns out that

$$
\Delta\left(B_{3}, B_{4}\right)=t t\langle b\rangle t t
$$

So the formula distinguishing $s_{1}$ from $s_{5}$ is

$$
\neg((t t\langle b\rangle t t)\langle a\rangle t t) .
$$

This formula explains why $s_{1}$ and $s_{5}$ are inequivalent because $s_{5}$ may engage in an $a-$ transition while in all the intermediate states (only $s_{5}$ here) a $b$-transition is available. This is not the case for state $s_{1}$. Note that this formula is minimal in the sense of definition 2.5 .

\section{Conclusions and Future Work}

This paper has shown how it is possible to alter the partition-refinement of Groote and Vaandrager for computing branching bisimulation equivalence to compute a formula in the Hennessy-Milner Logic with Until that distinguishes two inequivalent states. The generation of the formula relies on a postprocessing step that is invoked on a tree-based 

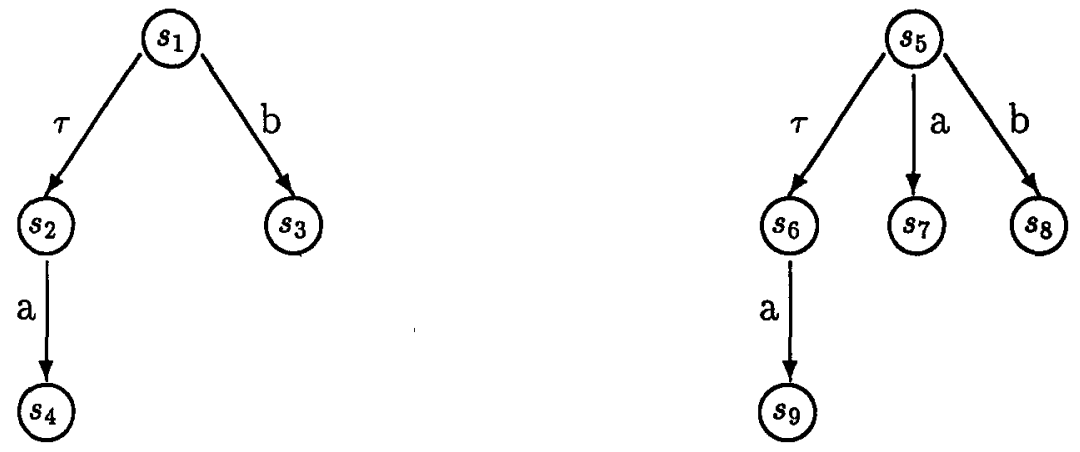

Figure 2: Two branching bisimulation inequivalent transition graphs.

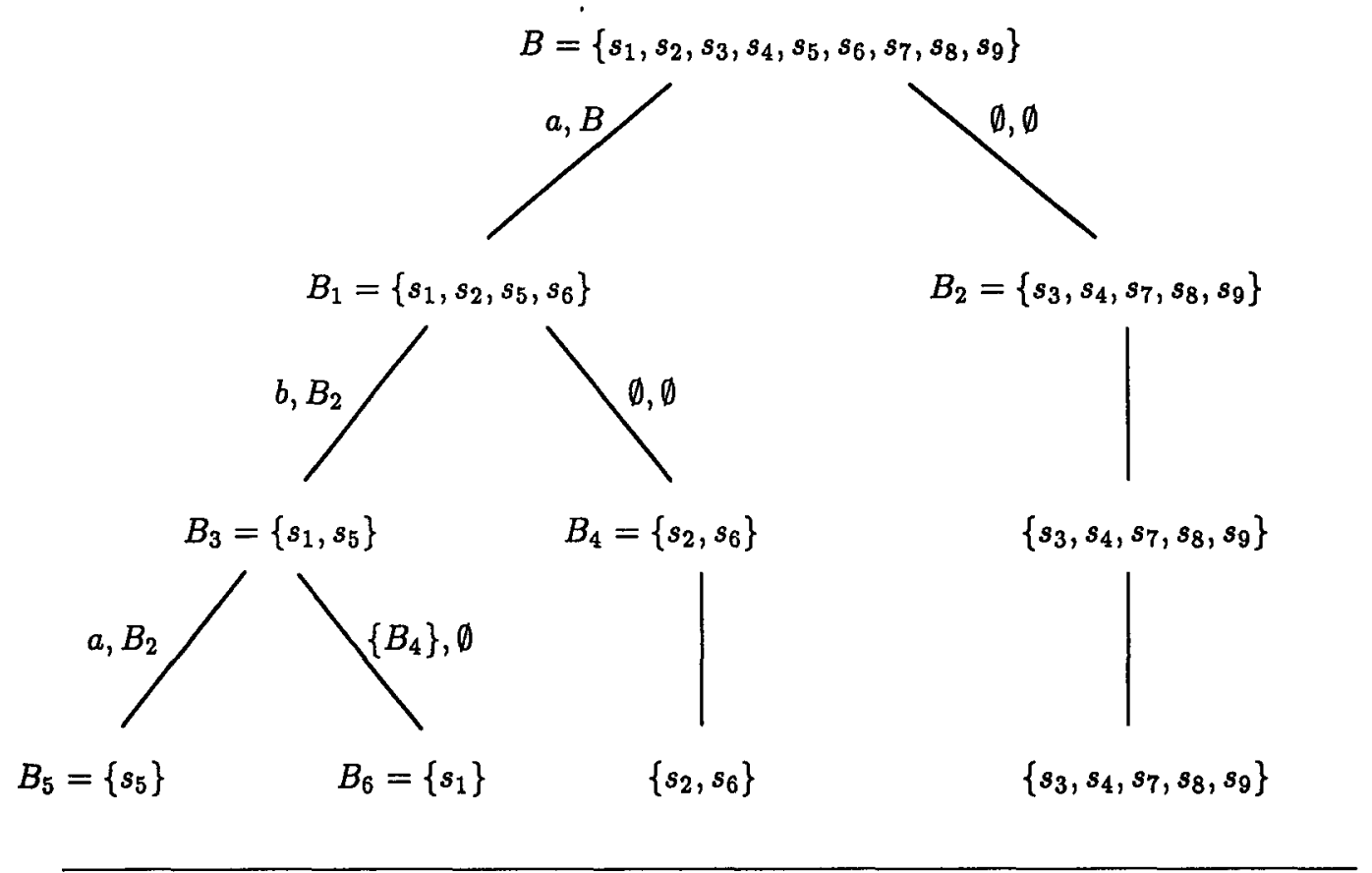

Figure 3: The generated tree of blocks. 
representation of the information computed by the equivalence algorithm. The postprocessing step has no effect on the worst-case complexity of the equivalence-checking algorithm, only the space complexity has changed slightly from $O(|\rightarrow|)$ to $O\left(|S|^{2}\right)$.

The most important direction for future work is tackling the problem of generating minimal formulas and moreover its complexity. Clearly, the complexity of the minimization procedure mentioned in passing at the end of section 3.3 needs to be analyzed fully; if this procedure is efficient enough, then it may be incorporated into the distinguishing formula generation procedure.

Another area of investigation would be an implementation of our technique, as an extension of the equivalence-checking algorithm of Groote and Vaandrager which is already implemented successfully.

\section{References}

[1] R. Cleaveland: On Automatically Distinguishing Inequivalent Processes. In Proceedings: 1990 Workshop on Computer-Aided Verification (R. Kurshan and E.M. Clarke, editors), DIMACS technical report 90-31, Vol. 2, New Yersey, 1990. To appear in Lecture Notes in Computer Science.

[2] R. DeNicola and F.W. Vaandrager: Three logics for branching bisimulation (extended abstract). In Proceedings $5^{\text {th }}$ Annual Symposium on Logic in Computer Science, Philadelphia, USA, pages 118-129, Los Alamitos, CA, 1990. IEEE Computer Society Press. Full version appeared as CWI Report CS-R9012.

[3] R.J. van Glabbeek: Comparative Concurrency Semantics and Refinement of Actions. $P h D$ thesis, Free University, Amsterdam, 1990.

[4] R.J. van Glabbeek and W.P. Weijland: Branching time and abstraction in bisimulation semantics (extended abstract). In G.X. Ritter, editor, Information Processing 89, pages 613-618. North-Holland, 1989.

[5] J.F. Groote and F.W. Vaandrager: An efficient algorithm for branching bisimulation and stuttering equivalence. In M.S. Paterson, editor, Proceedings $17^{\text {th }} I C A L P$, Warwick, volume 443 of $L N C S$, pages 626-638. Springer-Verlag, 1990.

[6] M. Hennessy and R. Milner: Algebraic Laws for Nondeterminism and Concurrency. Journal of the Association for Computing Machinery, v. 32, n. 1, pages 137-161, January 1985.

[7] P. Kanellakis and S.A. Smolka: CCS Expressions, Finite State Processes, and Three Problems of Equivalence. In Proceedings of the Second ACM Symposium on the Principles of Distributed Computing, 1983.

[8] H. Korver: The Current State of Bisimulation Tools. In report P9101, Programming Research Group, Univerisity of Amsterdam, 1991.

[9] H. Korver: Computing Distinguishing Formulas for Branching Bisimulation (Full Version). In report CS-R9121, CWI, Amsterdam, 1991.

[10] R. Milner: Communication and Concurrency. Prentice Hall, 1989. 